\title{
Direct shoot organogenesis in vitro from mature embryos of maize
}

Humud B.M.H.*, Yudakova O.I.

Saratov State University, Saratov, Russia

*e-mail:bobogold18@gmail.com

Biotechnological and genetic engineering methods can significantly accelerate the selection process. An important step in these methods is the regeneration of plants in an in vitro culture. Currently, considerable experience has been gained in the regeneration of maize plants through somatic embryogenesis (Santos et al., 1984; Alatortseva, 1994; Huang, Wei, 2004; Obert et al., 2004; Tang et al., 2006; Ahmadabadi et al., 2007; Rakshit et al., 2010; Joshi et al., 2014; etc.). However, regeneration through callus cultures is often accompanied by somaclonal variability, which is undesirable when cloning unique genotypes and carrying out genetic engineering studies. Unwanted variability can be avoided by plant regeneration through direct organogenesis. Currently, there is information about single successful attempts to induce direct organogenesis for some maize genotypes (Ahmad et al., 2017; Ovchinnikova et al., 2018; Humud et al., 2018). The aim of our study was the clonal micropropagation through direct organogenesis without callusogenesis of the three maize lines: KM (Brown marker), AT-TM (bm, wx, y) and AT-TM (lg, y). The lines AT-TM (bm, wx, y) and AT-TM (lg, y) was developed at the Saratov State University. Mature embryos were used as the primary explants. The conditions for the sterilization of the kernels were detected. For optimal sterilization of grains the "Domestos", $70 \%$ ethyl alcohol and $0.1 \%$ mercuric chloride were used. The different media for the initiation, micropropagation and rooting were tested. The best results were obtained on MS medium supplemented with $2.0 \mathrm{mg} / 1 \mathrm{BAP}$. The $3-5$ small axilary shoots developed in the basal parts of the explant after 3-4 weeks of cultivation. Microshoots were elongated on the MS medium supplemented with $0.2 \mathrm{mg} / 1$ BAP. Microshoots in the length $8-10 \mathrm{~mm}$ were rooted on the MS medium without hormones. Acknowledgements: This work was supported by the Ministry of Education and Science of the Russian Federation as part of the basic part of the state assignment in the field of scientific activity on assignment No. 6.8789.2017/BC. 